\title{
¿Cerca o lejos? Discursos y subjetividad en las relaciones entre el lugar de residencia y la movilidad
}

\author{
Daniel Orellana. Universidad de Cuenca, Cuenca, Ecuador. \\ Carla Hermida. Universidad del Azuay, Cuenca, Ecuador. \\ María-Augusta Hermida. Universidad de Cuenca, Cuenca, Ecuador.
}

RESUMEN | La relación entre forma urbana y transporte ha sido un tema ampliamente estudiado en las últimas décadas. No obstante, el vínculo entre las preferencias de residencia y los modos de desplazamiento de las personas no ha sido suficientemente estudiado en Latinoamérica, a pesar de ser un factor determinante de los patrones de expansión urbana. En el presente trabajo se investigó esta relación para el caso de Cuenca, Ecuador. Con tal fin, se aplicó la metodología Q, consistente en un conjunto de técnicas cuanti-cualitativas de análisis sistemático de la subjetividad. Se extrajeron y caracterizaron tres discursos predominantes en la ciudad en relación con preferencias de residencia y movilidad, que abordan diferentes perspectivas: un discurso orientado a la movilidad activa, otro orientado al transporte público, y finalmente uno orientado a la exclusividad y autosegregación. Esperamos que estudios como este contribuyan a la formulación de políticas que atiendan la diversidad de perspectivas de la población, y potencien las relaciones de proximidad.

PALABRAS CLAVE $\mid$ morfología urbana, transporte urbano, ciudades intermedias.

ABSTRACT | The relationship between urban form and transport has been an important research topic in recent decades. However, the relationship between preferences of location and typology of residence and transportation modes has not been sufficiently studied in Latin America, despite being a determining factor of urban expansion patterns. In the present work, we investigate this relationship in the city of Cuenca (Ecuador) using the $Q$ Methodology, a set of quantitative-qualitative techniques for the systematic analysis of subjectivity. We detected three predominant discourses characterising different perspectives: a) oriented to active mobility, b) oriented to public transport, and c) oriented to exclusivity and self-segregation. We expect that the present and further studies will contribute and inform public policies which address the diversity of perspectives of the population and enhance proximity relationships.

KEYWORDS | urban morphology, urban transportation, intermediate cities. 


\section{Introducción}

La relación dialéctica entre forma urbana y modos de viaje ha sido un tema de discusión en décadas recientes, y probablemente uno de los más estudiados en planificación urbana en los últimos diez años (Ewing \& Cervero, 2017). Este interés se debe, en parte, a la prominencia de la movilidad en las ciudades como uno de los problemas urbanos más prevalentes, y a la inherente complejidad de su tratamiento. Miralles-Guasch (2002) llama a la relación entre forma urbana y movilidad el "binomio imperfecto", y sostiene que hasta hace poco "su estudio se reducía a un análisis causal, donde cada uno de ellos era o bien el elemento causante o bien el consecuente" (p. 11). La autora sostiene, no obstante, que se debe mirar esta relación como bidireccional, es decir, que la morfología urbana afecta las formas de desplazamiento de los ciudadanos, y también viceversa: las estrategias de movilidad de las personas sostienen, modifican y generan formas urbanas.

Algunos investigadores exploran esta relación bidireccional en lo que se conocen como las "variables D": Densidad (de personas, viviendas, empleos), Diversidad (de usos, comunidades, actividades), Diseño (de calles, espacios públicos e infraestructura), Destinos (accesibilidad a fuentes de trabajo, comercios y servicios), y Distancia (a transporte público) (Ewing \& Cervero, 2010; Handy \& Xing, 2011). Estas variables $\mathrm{D}$ han sido ampliamente investigadas durante la última década, $\mathrm{y}$ aunque el consenso sobre su relación con el modo de transporte es generalizado, lo cierto es que los resultados reportados sobre las relaciones específicas son mixtos e incluso contradictorios, lo que dificulta llegar a conclusiones definitivas (Manville, 2017; Olaru et al., 2011; Stevens, 2017).

El interés que despierta este tema rebasa las fronteras disciplinares y, como consecuencia, el acervo de literatura existente es diverso y, en cierta forma, desorganizado (Handy, 2017). Varios factores contribuyen a esta variabilidad: diversidad de métodos de recolección y análisis de datos, falta de acuerdo en las métricas analizadas, diferencias entre los contextos sociales y culturales (Cervero et al., 2009), ausencia de modelos conceptuales (Handy, 2017), entre otros. Por ello, para entender mejor la relación entre forma urbana y elección del modo de transporte se requieren enfoques que vayan más allá de la modelación puramente estadística.

Si bien esta temática ha sido estudiada en Latinoamérica (Blanco, 2016; Figueroa, 2005; Gutiérrez, 2010), aún quedan por indagar las particularidades de diferentes tipos de ciudades; por ejemplo, las intermedias. En este contexto, el presente estudio explora los puntos de vista que tienen las personas sobre la relación del entorno y tipología del lugar de residencia con las preferencias de modos de viaje cotidiano, centrándose en la ciudad de Cuenca (Ecuador). A través de la aplicación del método Q (Watts \& Stenner, 2012) para el análisis de subjetividad, se extrajeron discursos representativos en las perspectivas de personas que residen en diferentes zonas de la ciudad. Los elementos constitutivos de los puntos de vista estuvieron relacionados con los medios de transporte disponibles y las características de los tejidos urbanos, condiciones que determinan la experiencia diaria y el comportamiento de los residentes. Los resultados permiten una mayor claridad sobre las perspectivas 
predominantes que influencian las decisiones de la población acerca del lugar de residencia y la elección del modo de transporte.

\section{Trabajos relacionados}

\section{Movilidad y forma urbana}

La forma urbana constituye la estructura espacial de la ciudad, que depende de las calles, bloques, lotes, edificios e infraestructura (Capel, 2002; de Solà-Morales i Rubió, 1997; Oliveira, 2016); del uso del suelo (Herold et al., 2002; Huang et al., 2007); de la topografía, los elementos naturales, los flujos de energía y materiales (Lynch, 1984); de los aspectos socioeconómicos, como el número de habitantes y la densidad de población (Frenkel \& Ashkenazi, 2008; Kasanko et al., 2006; Tsai, 2005); o de los procesos de transformación, como la construcción y el mercado (de Solà-Morales i Rubió, 1997). Sin embargo, en todos los casos, la forma urbana se refiere a las propiedades de la ciudad en un momento específico del tiempo. Coppola et al. (2014) han mostrado que las formas urbanas tienen diferencias en su grado de sostenibilidad, y parecería que el desarrollo urbano compacto es mejor que otras formas de desarrollo espacial. A pesar de esto, algunos críticos han sugerido que el desarrollo compacto podría implicar un aumento del nivel de congestión urbana (Cheshmehzangi \& Butters, 2016; Turok, 2016) y también un aumento de los precios de la vivienda, que en algunos casos crean exclusión social y segregación (Palm et al., 2014).

Varios autores coinciden en la relación intrínseca que existe entre la expansión urbana y el desarrollo de las tecnologías del transporte (automotores e infraestructuras) (Ewing \& Hamidi, 2015; Zhao \& Lu, 2011), sin desconocer otras variables que han provocado la expansión de las ciudades (políticas públicas, mercado inmobiliario, separación de usos de suelo, cambios en los niveles de ingreso y en la conformación familiar, etcétera). Li y Lai (2013) analizaron 36 ciudades en el mundo y encontraron seis tipos de formas urbanas que surgen de conectar el uso del suelo con el transporte. Los resultados son heterogéneos, evidenciando las particularidades de cada región, determinadas por las características socioeconómicas y preferencias culturales de la población. Esto explica, por ejemplo, por qué en ocasiones una buena infraestructura para cierto modo de viaje, no necesariamente garantiza una alta participación en dicho modo, resaltando la dependencia contextual de las soluciones (Klinger et al., 2013).

\section{Breve descripción del método $\mathrm{Q}$}

El método Q, propuesto por William Stephenson (1953), es un conjunto de principios psicológicos, estadísticos y de filosofía de la ciencia aplicados para analizar la subjetividad en las perspectivas de un grupo heterogéneo de personas acerca de un tema específico, y extraer los discursos predominantes (Watts \& Stenner, 2012). El método Q utiliza herramientas cualitativas y cuantitativas para realizar un estudio sistemático de la subjetividad implícita en las perspectivas de los participantes de un grupo, a partir de una técnica de ordenación de afirmaciones y de análisis factorial. Aunque el método Q tiene más de sesenta años, es poco conocido en la literatura de estudios de ciudad, por lo que se lo presenta brevemente en esta sección. Se puede 
encontrar un tratamiento más profundo del método y ejemplos de aplicación en el trabajo de Van Exel y De Graaf (2005). Para una revisión sistemática y un listado de los elementos clave que se requiere reportar en un estudio basado en el método Q, se puede consultar el trabajo de Dziopa y Ahern (2011). Además, para una explicación detallada de la técnica de bootstrap para el método Q se recomienda revisar Zabala y Pascual (2016).

En el método $\mathrm{Q}$, un grupo de participantes (conjunto $P$ ) recibe un juego de tarjetas con declaraciones que representan un amplio rango de puntos de vista (conjunto $Q$ ) sobre la temática de estudio y las ordena en una estructura piramidal (ordenación Q), según su grado de acuerdo individual (Figura 1). Durante el ejercicio, el investigador indaga y registra los razonamientos y motivaciones expresadas por el participante, para utilizarlos más adelante durante la etapa de interpretación. Las ordenaciones resultantes de todos los participantes se tabulan, asignándole a cada declaración un puntaje según su posición en la pirámide, y se calcula la matriz de correlación entre ordenaciones. A continuación, se realiza un análisis de factores de la matriz de correlación para identificar el número de agrupamientos naturales. Cada factor o agrupamiento natural resultante representa una perspectiva sobre el tema por tratar, y cada ordenación Q recibe un valor de "carga", que representa el nivel en el que se asocia con cada factor. Finalmente, el conjunto original de factores se rota de acuerdo con una función estadística (p.e., máxima varianza) o consideraciones teóricas del investigador para facilitar su interpretación. Cada factor del conjunto final representa un grupo de puntos de vista individuales que tienen alta correlación entre sí y baja correlación con otros. El paso final del análisis consiste en calcular los puntajes de factor y puntajes de diferencia. Estos puntajes permiten detectar declaraciones definitorias (que definen un factor), diferenciantes (que diferencian factores entre sí), y de consenso (compartidas por diferentes factores). Cada factor extraído puede ser interpretado como un discurso y representado en la misma estructura piramidal para facilitar la interpretación. Además, cada participante del conjunto P puede ser "cargado" en uno o más discursos en función del grado de similitud entre su ordenación y los factores extraídos.

Para interpretar los resultados del análisis, el investigador examina los factores extraídos y los analiza como discursos, prestando atención a las declaraciones que se encuentran en los extremos izquierdo y derecho de las pirámides, y a las declaraciones definitorias y diferenciantes. Además, las cargas de los participantes en los discursos permiten explorar posibles asociaciones entre las características de los participantes y de los discursos. Adicionalmente, el investigador examina las explicaciones y razonamientos de los participantes registrados durante la ordenación como una guía para interpretar, verificar e ilustrar los discursos. Los discursos resultantes suelen ser nombrados con etiquetas o nombres representativos.

Aunque el método Q tiene varias décadas, su componente analítico ha tenido pocas innovaciones, y la confiabilidad de sus resultados ha sido puesta en duda ocasionalmente. Por ejemplo, no se conoce si el resultado de un ejercicio podría haber variado en el caso de que uno o más miembros del grupo hubieran sido reemplazados. Recientemente, Zabala y Pascual (2016) propusieron una técnica de bootstrapping para determinar la estabilidad y confiabilidad de los discursos extraídos, así 
como para mejorar su interpretación. En la técnica propuesta, se aplica $n$ veces un remuestreo con reemplazo al conjunto de Ordenaciones $\mathrm{Q}$, realizando el análisis completo (extracción de factores, asignación de cargas a los factores) para cada remuestreo. Los resultados de todos los remuestreos representan una estimación de la distribución de cada una de las medidas obtenidas (por ejemplo, los puntajes de factor), que puede ser analizada con estadísticos de centralidad y dispersión (por ejemplo, media y desviación estándar). Estas medidas son estimaciones alternativas de la muestra original.

FIGURA I | Ejemplo de plantilla para la ordenación Q

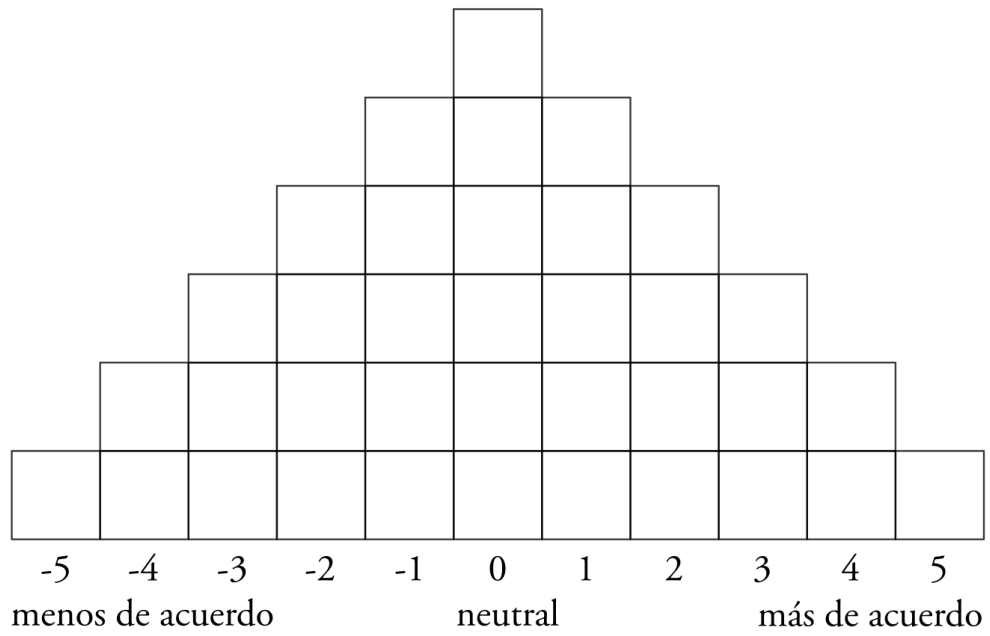

FUENTE: ELABORACIÓN PROPIA

\section{Método Q y movilidad}

En el campo de la movilidad se ha aplicado el método Q para entender las actitudes, preferencias, percepciones y subjetividades detrás de las decisiones (Hickman \& Vecia, 2016; Jones et al., 2012; Rajé, 2007; Van Exel et al., 2011), para establecer relaciones entre medioambiente y movilidad (Morinière \& Hamza, 2012), o para evaluar la aceptación ciudadana a políticas de transporte (Cools et al., 2009). Otros investigadores lo han usado para estudiar las opiniones y preferencias con respecto al barrio (Borth \& Summers, 2018; Van Dijk et al., 2015), o a entornos específicos del vecindario (Annear et al., 2009).

El objetivo último del método $Q$ es encontrar discursos predominantes. Por ejemplo, en el estudio de Rajé (2007) se encontraron cuatro líneas discursivas: el residente contento, el usuario del vehiculo como un escape por las deficiencias locales, el defensor de la multimodalidad y el teórico descontento con los problemas del transporte y que cree teóricamente en la participación ciudadana. Cools et al. (2012) detectaron también cuatro discursos sobre la opinión acerca de las políticas públicas: aquellos viajeros que apoyan las medidas de reducción de velocidad, aquellos en 
contra, los viajeros que están a favor de políticas de restricción de la demanda, y aquellos opuestos a medidas innovadoras. Morinière y Hamza (2012) aplicaron el instrumento a expertos para explorar la percepción sobre la relación entre ambiente y movilidad, y encontraron también cuatro discursos: los humanistas determinados, los pragmáticos benévolos, los proteccionistas cínicos y los realistas críticos. En varios estudios las líneas discursivas resultantes revelaron fuertes inclinaciones hacia uno $\mathrm{u}$ otro modo de desplazamiento (bicicleta, a pie, vehículo privado, transporte público) (Cools et al., 2009; Hickman et al., 2018; Hickman \& Vecia, 2016; Jones et al., 2012; Van Exel et al., 2011).

Varios estudios con método Q se han enfocado en las preferencias del lugar de residencia. Borth y Summers (2018) segmentaron a los compradores de bienes inmuebles por su preferencia de localización, encontrando tres líneas discursivas: el nuevo habitante suburbano, el habitante suburbano maduro y el habitante consciente de la importancia de la localización. Algunos estudios exploraron las preferencias del lugar de residencia de adultos mayores y descubrieron discursos relacionados con la predilección por un lugar de calma, la necesidad de equipamientos, y la posibilidad de interacción social (Annear et al., 2009), así como relacionados con la accesibilidad y la seguridad (Van Dijk et al., 2015).

En la literatura revisada surgen algunas similitudes y diferencias en las decisiones metodológicas que han servido de guía para el presente estudio. Mientras que el tamańo del conjunto Q y el número de participantes en el conjunto P es variable, los discursos representativos extraídos se mantienen siempre entre tres y cuatro. La definición del conjunto P se estratifica, por lo general, en función de edad y género, junto a otros criterios específicos de cada proyecto. Los trabajos revisados han sido llevados a cabo mayoritariamente en países desarrollados: Reino Unido (Hickman \& Vecia, 2016; Jones et al., 2012; Rajé, 2007), Estados Unidos (Van Exel et al., 2011), Bélgica (Cools et al., 2009; Cools et al., 2012), Canadá (Borth \& Summers, 2018) y Holanda (Van Dijk et al., 2015). La presente investigación representa, por lo tanto, el primer ejemplo de la exploración de la relación entre movilidad y forma urbana utilizando el método $Q$ en una ciudad latinoamericana. En este sentido, se pretende aportar a disminuir la brecha entre los conceptos globales y las realidades regionales al momento de abordar las temáticas urbanas (Dobbs et al., 2019).

\section{Metodología}

\section{Área de estudio}

Cuenca es una ciudad intermedia de aproximadamente 400.000 habitantes en los Andes Sur del Ecuador. Tiene un clima húmedo de montaña caracterizado por temperaturas templadas y precipitaciones distribuidas a lo largo del año, con una época seca entre julio y septiembre. El centro histórico de la ciudad, declarado Patrimonio Cultural de la Humanidad en 1999, constituyó el límite urbano hasta la década de los sesenta. Desde entonces, la ciudad comenzó a experimentar un rápido crecimiento físico debido a un plan basado en los principios del urbanismo moderno, el cual fue generando diferentes tipos de tejidos urbanos que, a manera de hipótesis, consideramos que están relacionados con la movilidad. Definimos el tejido urbano 
como un área homogénea de la ciudad, caracterizada por similitudes en los patrones y diseńos de calles y manzanas, por la manera en que se dividen y organizan los predios, la forma y escala de los lotes, el diseño de vías y espacios de estacionamiento, y las relaciones entre el paisaje mineral y vegetal (Wheeler, 2015). En la ciudad de Cuenca existe actualmente un total de 17 tejidos urbanos (Hermida et al., 2019). Para esta investigación se analizaron las percepciones sobre los diez más representativos (Figura 2). Aquellos que datan de la fundación de la ciudad y los que han sido parte de procesos de lotización previos a los años setenta, se caracterizan por tener densidades adecuadas, buena conectividad y espacio público. Tal es el caso del damero urbano, cuasi damero, damero de cuadras rectangulares y las villas ajardinadas. A partir de los años setenta, el modelo neoliberal imperante en la región llevó a transformaciones urbanas no sustentables y a una expansión urbana descontrolada, por lo que aparecen tejidos como los denominados en proceso de consolidación tanto dentro como fuera del límite urbano, los de expansión rural, los caminos rurales y las urbanizaciones (cerradas), donde la planificación llegó tarde o nunca. Entre estos dos extremos, se encuentra el tejido orgánico, que cumple con características de densidad y diversidad propias de las áreas más consolidadas, pero cuyos patrones de calles son irregulares y, por ende, presenta una conectividad moderada.

El Municipio de Cuenca asumió las competencias de planificación y regulación de transporte a partir del año 1999, creando el Plan de Tráfico Sustentable 19992000. Este plan tuvo varias actualizaciones y transformaciones a lo largo de los siguientes años, hasta que en 2012 se planteó el reemplazo de la línea troncal de autobuses por una troncal tranviaria. El proyecto del tranvía no estuvo libre de fuertes cuestionamientos técnicos, económicos y políticos; a pesar de ello, inició su operación con pasajeros en mayo del 2020. También destacan otros proyectos, como el sistema de bicicletas públicas que, a pesar de su limitada extensión, resultó un hito interesante para la movilidad activa de la ciudad.

Según el Plan de Movilidad y Espacios Públicos de la ciudad (Municipalidad de Cuenca, 2015), el vehículo privado es el principal medio de viaje para el 32,3\% de la población; el autobús lo es para el 30,7\%; y la caminata para el 30,4\%, lo cual sería indicativo de un sistema de movilidad relativamente sostenible. No obstante, el mismo documento indica que el $66 \%$ de la población anhela poder movilizarse en vehículo particular. El $75 \%$ de los desplazamientos se realiza en el interior del límite urbano, un 15\% entre el interior y el exterior, y el 10\% restante entre zonas rurales. El 33\% de viajes tiene lugar por motivos de trabajo, mientras que el estudio, las compras y las gestiones personales representan el 18\%, 17\% y 16\% de viajes, respectivamente. La mayor parte de desplazamientos a pie son de menos de 5 minutos, la mayoría de los viajes en autobús toman entre 6 y 15 minutos, y en vehículo entre 16 y 30 minutos.

Para el presente estudio, la ciudad ha sido dividida en tres grandes zonas (Figura 2): la zona central, que incluye el área de la ciudad delimitada por la Av. de las Américas y la Circunvalación Sur; la zona urbana, que abarca el área alrededor de la zona central hasta la demarcación oficial del perímetro urbano de la ciudad; y, finalmente, el área de influencia inmediata definida en el Plan de Ordenamiento Territorial del cantón Cuenca. 
FIGURA 2 | Área de estudio. Las letras representan los principales tipos de tejidos urbanos

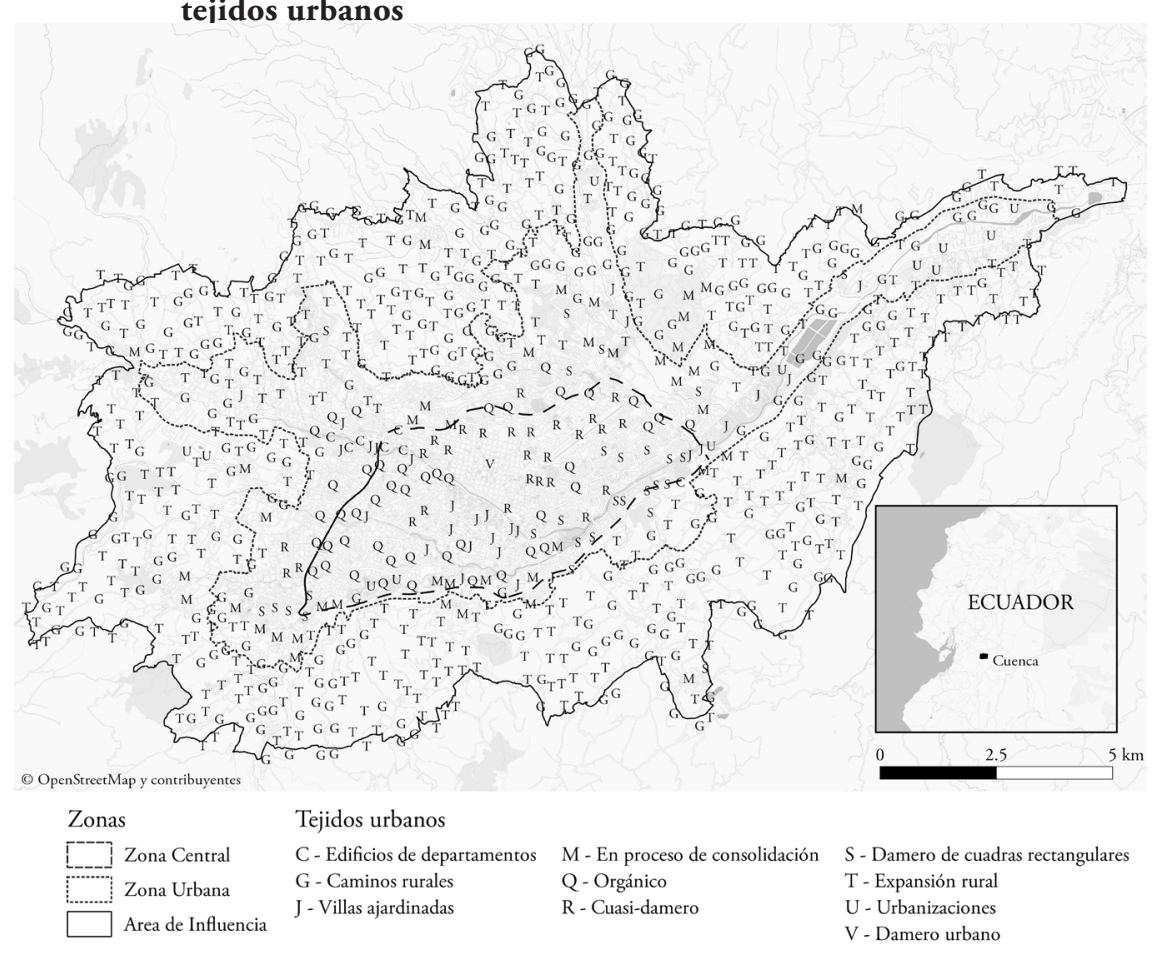

FUENTE: OPENSTREETMAP Y CONTRIBUYENTES. ELABORACIÓN PROPIA

\section{Creación del conjunto $\mathrm{Q}$}

Para la presente investigación se creó un concurso con declaraciones referidas a sus dos componentes temáticos: i) las preferencias sobre el lugar de residencia según las características de los tejidos urbanos seleccionados; y ii) las preferencias relacionadas con los modos de desplazamiento. Las declaraciones iniciales fueron producto de la revisión de literatura y de ejercicios en proyectos anteriores. Ya que el estudio busca detectar preferencias y anhelos, las frases fueron escritas en tiempo condicional. Las frases fueron revisadas y agrupadas para formar un conjunto Q con 36 declaraciones (Tabla 1) y posteriormente validadas por investigadores externos al proyecto. 
TABLA I | Conjunto Q

\begin{tabular}{|c|c|}
\hline ID & DECLARACIÓN \\
\hline D01 & Preferiría caminar para realizar mis actividades diarias. \\
\hline D02 & Creo que sería muy importante contar con patio o jardín propio. \\
\hline D03 & Preferiría un barrio en el que viva mucha gente. \\
\hline D04 & $\begin{array}{l}\text { Para mí, sería muy importante la existencia de veredas anchas en buen estado dentro de mi } \\
\text { barrio. }\end{array}$ \\
\hline D05 & Preferiría vivir en un barrio en el que existan comercios y servicios, pero no demasiados. \\
\hline D06 & $\begin{array}{l}\text { Me agradaría vivir en un conjunto habitacional cerrado con casas diferentes y espacio verde } \\
\text { propio. }\end{array}$ \\
\hline D07 & Para mí sería muy importante contar con parques cerca de mi casa. \\
\hline D08 & $\begin{array}{l}\text { Preferiría un barrio que no tenga muchas calles conectadas, me gustaría inclusive que existan } \\
\text { calles sin salida. }\end{array}$ \\
\hline D09 & Me agradaría vivir en un edificio de más de seis pisos. \\
\hline D10 & Me agradaría salir de mi casa y poder encontrar un taxi con facilidad. \\
\hline D11 & Para mí sería fundamental contar con garaje en mi predio. \\
\hline D12 & Me gustaría que mi casa sea pegada a la del vecino y no tenga retiro frontal. \\
\hline D13 & Preferiría vivir frente a vías principales y/o avenidas. \\
\hline D14 & Preferiría usar bus para llegar a mi trabajo o lugar de estudio. \\
\hline D15 & Me gustaría vivir en el campo. \\
\hline D16 & Me gustaría que existan muchos comercios, servicios y equipamientos cerca de mi casa. \\
\hline D17 & Me gustaría trasladarme en moto hacia mis diferentes lugares de destino. \\
\hline D18 & Preferiría vivir en departamento antes que en casa. \\
\hline D19 & Preferiría un barrio con todas las calles asfaltadas, destinadas al vehículo y moto exclusivamente. \\
\hline D20 & Creo que sería muy importante poder parquear en la vía del frente de mi casa. \\
\hline D21 & Me agradaría que mi casa esté separada de los vecinos en todos sus lados. \\
\hline D22 & Me interesaría vivir en un lugar cercano al centro histórico. \\
\hline D23 & Me gustaría poder utilizar bicicleta para trasladarme de un lugar a otro. \\
\hline D24 & $\begin{array}{l}\text { Me interesaría vivir en un barrio netamente residencial sin variedad de equipamientos } \\
\text { ni comercios. }\end{array}$ \\
\hline D25 & $\begin{array}{l}\text { Me parecería lindo un barrio que tenga casas grandes, pequeńas, edificios de departamentos, o } \\
\text { sea una variedad. }\end{array}$ \\
\hline D26 & Preferiría desplazarme en taxi para realizar mis actividades cotidianas. \\
\hline D27 & Me agradaría que existan ciclovías en mi barrio. \\
\hline D28 & Me interesaría vivir en un conjunto habitacional cerrado con las casas iguales entre ellas. \\
\hline D29 & Me gustaría una casa pegada a la del vecino con un pequeño retiro frontal. \\
\hline D30 & Preferiría utilizar auto privado para desplazarme diariamente. \\
\hline D31 & Me encantaría que pasen varias líneas de bus por mi barrio. \\
\hline D32 & Me interesaría que todas las vías de mi barrio estén conectadas. \\
\hline D33 & Preferiría un barrio en el que viva gente, pero no demasiada. \\
\hline D34 & Me gustaría vivir en una edificación patrimonial con patio interior. \\
\hline D35 & Me interesaría que mi casa esté frente a una calle no muy ancha. \\
\hline D36 & Preferiría ir de acompañante antes que de conductor. \\
\hline
\end{tabular}

FUENTE: ELABORACIÓN PROPIA 


\section{Creación del conjunto $P$}

El conjunto P se generó mediante una estratificación basada en la edad, el género, el nivel socioeconómico y el lugar de residencia según la zonificación predefinida. Con estos criterios se identificó y contactó a los potenciales participantes utilizando una técnica de bola de nieve. Se logró concretar la participación de 49 personas distribuidas en las diferentes categorías (Tabla 2). El nivel socioeconómico fue inicialmente estimado por los investigadores y luego corroborado durante la aplicación del instrumento con un sistema de clasificación. La participación de personas de estratos socioeconómicos bajos y de adultos mayores fue un reto importante debido a la utilización de redes sociales electrónicas para el reclutamiento. Esto fue parcialmente mitigado gracias a la técnica de bola de nieve.

TABLA 2 Características de los participantes del conjunto $P$

\begin{tabular}{|c|c|c|c|c|c|c|c|}
\hline CódIGo & ZONA & GÉNERO & EDAD & OCUPACIÓN & $\begin{array}{c}\text { MODO } \\
\text { PRINCIPAL DE } \\
\text { TRANSPORTE* }^{*}\end{array}$ & HIJOS & $\begin{array}{l}\text { CLASIFICACIÓN } \\
\text { ESTRATO SOCIO- } \\
\text { ECONÓMICO }\end{array}$ \\
\hline P01 & Central & $\mathrm{F}$ & $18-25$ & Trabajador & Bus & Sí & Bajo \\
\hline P02 & Central & $\mathrm{F}$ & $18-25$ & Estudiante & VPC & No & Medio \\
\hline P03 & Central & $\mathrm{F}$ & $18-25$ & Estudiante & Bicicleta & No & Alto \\
\hline P04 & Urbana & $\mathrm{F}$ & $18-25$ & Estudiante & VPC & No & Medio \\
\hline P05 & Urbana & $\mathrm{F}$ & $18-25$ & Estudiante & VPC & No & Alto \\
\hline P06 & Influencia & $\mathrm{F}$ & $18-25$ & Desempleado & Bus & Sí & Bajo \\
\hline P07 & Influencia & $\mathrm{F}$ & $18-25$ & Trabajador & VPC & Sí & Medio \\
\hline P08 & Influencia & $\mathrm{F}$ & $18-25$ & Trabajador & VPC & No & Alto \\
\hline P09 & Central & $\mathrm{F}$ & $26-65$ & Desempleado & Bus & Sí & Bajo \\
\hline P10 & Central & $\mathrm{F}$ & $26-65$ & Trabajador & VPC & Sí & Medio \\
\hline P11 & Central & $\mathrm{F}$ & $26-65$ & Trabajador & VPC & No & Alto \\
\hline P12 & Urbana & $\mathrm{F}$ & $26-65$ & Trabajador & Bus & Sí & Bajo \\
\hline P13 & Urbana & $\mathrm{F}$ & $26-65$ & Trabajador & Bus & Sí & Medio \\
\hline P14 & Urbana & $\mathrm{F}$ & $26-65$ & Trabajador & VPC & Sí & Alto \\
\hline P15 & Influencia & $\mathrm{F}$ & $26-65$ & Trabajador & Bus & Sí & Bajo \\
\hline P16 & Influencia & $\mathrm{F}$ & $26-65$ & Trabajador & VPP & Sí & Medio \\
\hline P17 & Influencia & $\mathrm{F}$ & $26-65$ & Trabajador & TC & Sí & Medio \\
\hline P18 & Influencia & $\mathrm{F}$ & $26-65$ & Trabajador & Bus & Sí & Medio \\
\hline P19 & Influencia & $\mathrm{F}$ & $26-65$ & Trabajador & VPC & Sí & Alto \\
\hline P20 & Central & $\mathrm{F}$ & $>65$ & Desempleado & VPP & No & Medio \\
\hline P21 & Central & $\mathrm{F}$ & $>65$ & Jubilado & VPP & Sí & Medio \\
\hline P22 & Central & $\mathrm{F}$ & $26-65$ & Jubilado & VPC & Sí & Alto \\
\hline P23 & Urbana & $\mathrm{F}$ & $>65$ & Desempleado & Bus & Sí & Bajo \\
\hline P24 & Urbana & $\mathrm{F}$ & $>65$ & Jubilado & VPC & No & Medio \\
\hline P25 & Influencia & $\mathrm{F}$ & $>65$ & Jubilado & VPP & No & Alto \\
\hline P26 & Central & $M$ & $18-25$ & Trabajador & VPC & No & Medio \\
\hline $\mathrm{P} 27$ & Central & $\mathrm{M}$ & $18-25$ & Estudiante & Bicicleta & No & Alto \\
\hline P28 & Urbana & $M$ & $18-25$ & Estudiante & Moto & No & Medio \\
\hline P29 & Urbana & $M$ & $18-25$ & Trabajador & VPC & No & Alto \\
\hline P30 & Influencia & $\mathrm{M}$ & $18-25$ & Estudiante & VPP & No & Medio \\
\hline $\mathrm{P} 31$ & Influencia & $\mathrm{M}$ & $18-25$ & Trabajador & VPC & No & Alto \\
\hline
\end{tabular}




\begin{tabular}{|c|c|c|c|c|c|c|c|}
\hline CóDIGO & ZONA & GÉNERO & EDAD & OCUPACIÓN & $\begin{array}{c}\text { MODO } \\
\text { PRINCIPAL DE } \\
\text { TRANSPORTE* }^{*}\end{array}$ & HIJOS & $\begin{array}{l}\text { CLASIFICACIÓN } \\
\text { ESTRATO SOCIO- } \\
\text { ECONÓMICO }\end{array}$ \\
\hline P32 & Central & $\mathrm{M}$ & $26-65$ & Estudiante & Bicicleta & No & Bajo \\
\hline P33 & Central & M & $26-65$ & Trabajador & Bus & Sí & Medio \\
\hline P34 & Central & M & $26-65$ & Trabajador & Bus & Sí & Medio \\
\hline P35 & Central & M & $26-65$ & Trabajador & Bus & No & Alto \\
\hline P36 & Central & $\mathrm{M}$ & $26-65$ & Trabajador & VPC & No & Alto \\
\hline P37 & Urbana & $\mathrm{M}$ & $26-65$ & Trabajador & Bus & Sí & Bajo \\
\hline P38 & Urbana & M & $26-65$ & Trabajador & VPC & No & Medio \\
\hline P39 & Urbana & M & $26-65$ & Trabajador & VPC & No & Medio \\
\hline $\mathrm{P} 40$ & Urbana & $\mathrm{M}$ & $26-65$ & Trabajador & VPC & Sí & Alto \\
\hline $\mathrm{P} 41$ & Influencia & $\mathrm{M}$ & $26-65$ & Trabajador & Caminata & Sí & Bajo \\
\hline $\mathrm{P} 42$ & Influencia & $\mathrm{M}$ & $26-65$ & Trabajador & VPC & Sí & Medio \\
\hline $\mathrm{P} 43$ & Influencia & $\mathrm{M}$ & $26-65$ & Trabajador & Bus & Sí & Medio \\
\hline P44 & Influencia & $\mathrm{M}$ & $26-65$ & Trabajador & VPC & Sí & Alto \\
\hline $\mathrm{P} 45$ & Central & $\mathrm{M}$ & $26-65$ & Jubilado & Bus & No & Medio \\
\hline $\mathrm{P} 46$ & Urbana & $\mathrm{M}$ & $>65$ & Jubilado & VPC & Sí & Alto \\
\hline $\mathrm{P} 47$ & Urbana & $\mathrm{M}$ & $>65$ & Jubilado & VPC & No & Medio \\
\hline P48 & Urbana & M & $>65$ & Jubilado & VPC & No & Alto \\
\hline P49 & Influencia & M & $>65$ & Trabajador & VPC & Sí & Alto \\
\hline
\end{tabular}

* VPC= VEHÍCULO PRIVADO (CONDUCTOR), VPP= VEHÍCULO PRIVADO (PASAJERO), TC= TAXI O CAMIONETA DE ALQUILER

FUENTE: ELABORACIÓN PROPIA

\section{Aplicación}

Los investigadores fijaron una cita previa con cada uno de los participantes, explicando el motivo del proyecto y la duración aproximada del ejercicio (una hora). No existió un incentivo por la participación, pero se enviaron mensajes posteriores de agradecimiento a los voluntarios. Durante la aplicación, el investigador explicó detalladamente la técnica e introdujo el ejercicio con el siguiente enunciado: "Este estudio es para medir sus preferencias acerca del barrio donde le gustaría vivir y la forma de desplazamiento que usted preferiría. Lea los siguientes enunciados y ordénelos según cuán de acuerdo esté con cada uno de ellos. No importa el barrio en el que resida o el medio de transporte que utilice actualmente, sino cuál sería el de su preferencia”. A continuación, el participante procedió a una primera ordenación de las tarjetas en tres grupos: ("de acuerdo", "en desacuerdo", "indiferente"). A partir de estos tres grupos, el participante procedió a la ordenación final de las afirmaciones en la plantilla piramidal. Al finalizar el ordenamiento, se realizaron las siguientes preguntas: ¿Qué características tiene el barrio de su preferencia? ¿Por qué lo prefiere? ¿Cuál es el medio de transporte de su preferencia? Y ¿por qué lo prefiere? Las respuestas se grabaron en audio para su posterior transcripción verbatim y análisis.

\section{Análisis}

Los datos de las ordenaciones Q y del conjunto P se almacenaron en tablas .csv y se analizaron utilizando el software $\mathrm{R}$ (R Core Team, 2016) con el paquete qmethod (Zabala, 2014). Se realizó un gráfico de sedimentación y se determinó que, a partir 
del quinto factor, la varianza explicada no superaba el 5\% (Figura 3) y solo tenía una ordenación cargada en él, por lo que solamente se analizaron las características de modelos con 2, 3 y 4 factores.

\section{FIGURA 3 | Varianza explicada por los 10 primeros factores}

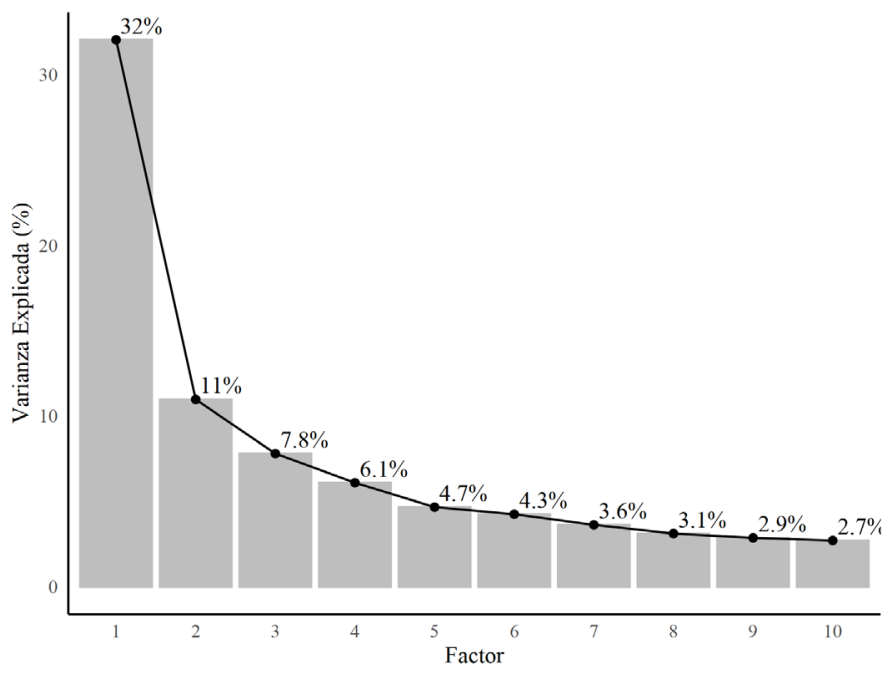

FUENTE: ELABORACIÓN PROPIA

Además, se utilizó la técnica de bootstrapping para determinar la estabilidad y confiabilidad de los factores extraídos, así como mejorar su interpretación (Zabala \& Pascual, 2016). Una inspección de los modelos permitió identificar el de 3 factores como el más estable y el que permitía una diferenciación más clara y un mayor valor interpretativo de los factores extraídos. Los factores resultantes del modelo seleccionado fueron representados en tablas y gráficos que permitieron distinguir las declaraciones definitorias, diferenciantes y de consenso. Finalmente, se elaboraron descripciones de los discursos representados por cada factor y se asignó un nombre representativo a cada uno. El código fuente y los resultados detallados están disponibles de forma pública en un repositorio online <https://llactalab.ucuenca.edu.ec/ investigacion/mas-alla-del-petroleo/>.

\section{Resultados}

\section{Resultados empíricos}

Luego de analizar las características de los modelos con 2, 3 y 4 factores, se seleccionó el modelo con 3 factores para el análisis, ya que representa un balance entre simplicidad y poder explicativo. En este modelo, 45 de los 49 participantes estuvieron cargados en al menos un factor, es decir, que los factores extraídos son útiles para representar los puntos de vista integrados de la gran mayoría de participantes. 
El método de bootstrapping identificó a 33 participantes que fueron marcados al menos un $75 \%$ de veces en alguno de los factores. En este modelo, todos los factores tuvieron autovalores mayores a 7,8, indicando que capturan una alta varianza; los valores de la confiabilidad compuesta fueron mayores a 0,98 y los errores estándar de los puntajes fueron menores a 0,14 (Tabla 3).

TABLA 3 | Características del modelo con 3 factores

\begin{tabular}{|l|c|c|c|}
\cline { 2 - 4 } \multicolumn{1}{c|}{} & FI & F2 & F3 \\
\hline Número de participantes cargados & 13 & 17 & 16 \\
\hline Autovalores & 8,800 & 8,299 & 7,808 \\
\hline Porcentaje de varianza explicada & 17,958 & 16,938 & 15,934 \\
\hline Confiabilidad compuesta & 0,981 & 0,986 & 0,985 \\
\hline Error estándar de los puntajes de factor & 0,137 & 0,120 & 0,124 \\
\hline
\end{tabular}

FUENTE: ELABORACIÓN PROPIA

Con el fin de facilitar la interpretación del discurso correspondiente a cada factor, se representa en la Figura 4 el puntaje $Z$ de cada declaración en cada uno de los factores extraídos mediante la técnica de bootstrapping. La posición de los símbolos en el eje horizontal, con respecto a la línea de 0 , indican si el discurso está de acuerdo (puntaje positivo) o en desacuerdo con la declaración (puntaje negativo). Además, la distancia entre los símbolos de los factores en una declaración indica el grado de coincidencia. Adicionalmente, los errores estándar derivados de la fase de bootstrapping se ilustran como líneas grises para estudiar la estabilidad y confiabilidad de los resultados, indicando el rango en el que se podría ubicar el puntaje Z; las barras más largas, por lo tanto, serán indicativas de que el resultado puntual es inestable. Siguiendo las recomendaciones de Zabala y Pascual (2016), se identificaron las declaraciones que tienen alta prominencia (valor absoluto del puntaje $Z$ mayor a 1) y alta estabilidad (error estándar menor a 0,4 ), obteniendo un conjunto de 10 declaraciones con alto poder interpretativo. De manera similar, las cargas de los participantes en los factores extraídos se representan en la Figura 5, donde se han resaltado con doble línea los participantes que han sido cargados más del 75\% de las veces en un discurso durante la fase de bootstrapping. 


\section{Figura 4 | Puntajes Z de las declaraciones en cada discurso (técnica de bootstrapping)}

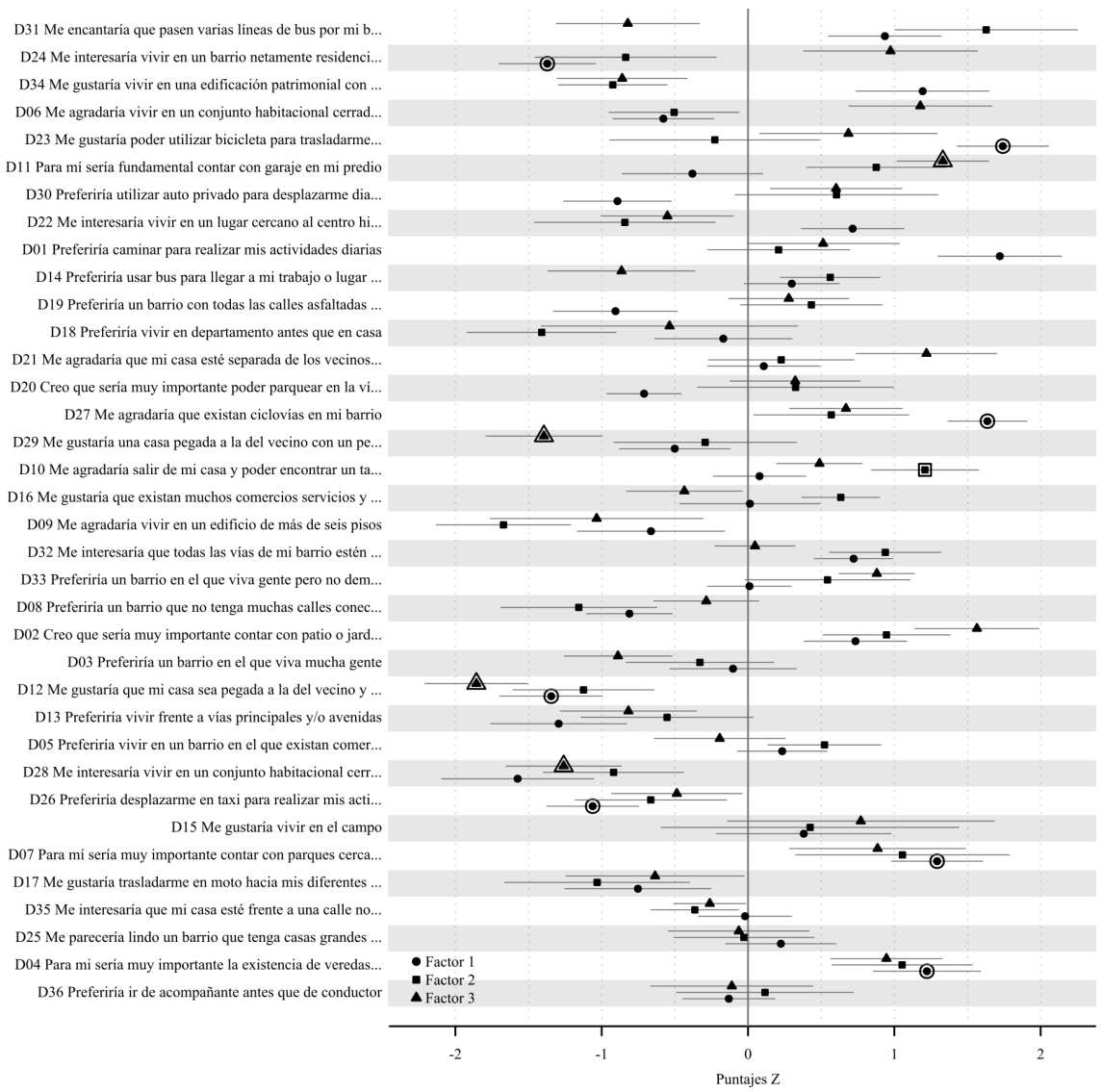

NOTA: LOS PUNTOS REPRESENTAN EL VALOR MEDIO DEL PUNTAJE Z DE CADA DECLARACIÓN PARA CADA FACTOR EXTRAÍDO. LAS LÍNEAS REPRESENTAN LOS ERRORES ESTÁNDAR. LOS SÍMBOLOS CON DOBLE CONTORNO INDICAN ALTO PODER INTERPRETATIVO

FUENTE: ELABORACIÓN PROPIA 
FIGURA 5 | Cargas de los participantes en cada discurso (técnica de bootstrapping)

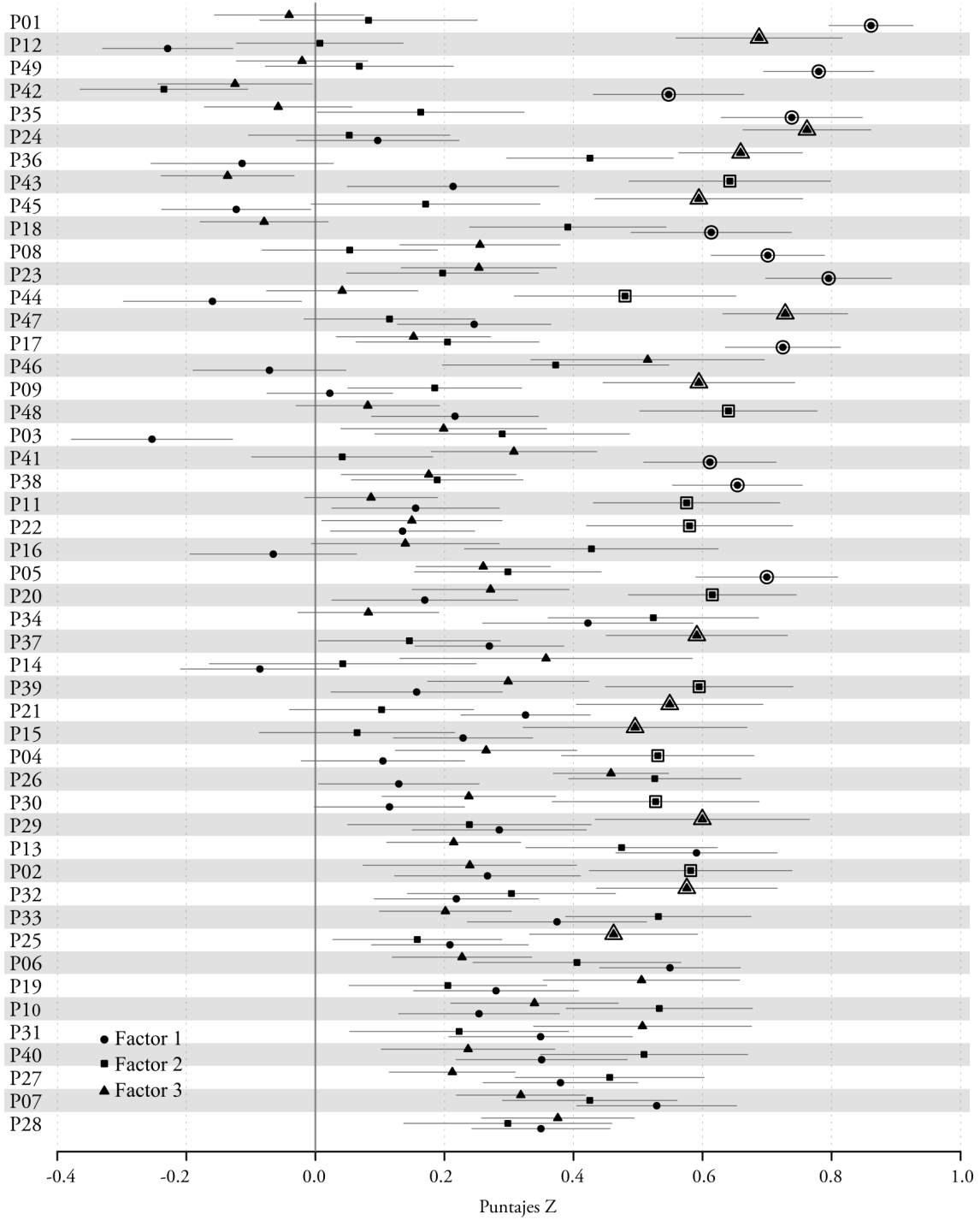

NOTA: LOS PUNTOS REPRESENTAN EL VALOR MEDIO DEL PUNTAJE Z DE CADA PARTICIPANTE EN CADA FACTOR EXTRAÍDO. LAS LÍNEAS REPRESENTAN LOS ERRORES ESTÁNDAR. LOS SÍMBOLOS CON DOBLE CONTORNO INDICAN QUE LA CARGA FUE SIGNIFICATIVA

FUENTE: ELABORACIÓN PROPIA 


\section{Discursos descubiertos}

Del análisis anterior surgen tres tipos de discurso claramente identificables: i) Orientados a la movilidad activa; ii) Orientados al transporte público; y iii) Orientados a la exclusividad y autosegregación. A continuación, detallamos las características principales de cada uno de ellos.

\section{Discurso 1: Orientados a la movilidad activa}

En este discurso, la movilidad es más importante que la tipología de vivienda o el tejido urbano al momento de seleccionar el lugar de residencia. Se expresa en un fuerte deseo de vivir en un lugar que permita la movilidad activa y la conectividad; se valoran veredas y verde urbano; se rechazan tejidos urbanos que dificultan el uso de modos no motorizados, como el condominio cerrado y la localización en la periferia. Este discurso coincide fuertemente con declaraciones que se refieren principalmente a la movilidad activa: "Me gustaría poder utilizar bicicleta para trasladarme de un lugar a otro" (D23), "Me agradaría que existan ciclovías en mi barrio" (D27), "Preferiría caminar para realizar mis actividades diarias" (D01). Otras dos declaraciones de fuerte acuerdo están relacionadas con la infraestructura para la caminata y la recreación: "Para mí sería muy importante contar con parques cerca de mi casa" (D7); y "Para mí, sería muy importante la existencia de veredas anchas en buen estado dentro de mi barrio" (D4). Sin embargo, en estas declaraciones hay poca diferenciación con otros discursos; es decir, que las tres perspectivas coinciden en la importancia de estos elementos. Finalmente, también está muy de acuerdo con una tipología de vivienda específica: "Me gustaría vivir en una edificación patrimonial con patio interior" (D34).

Por otro lado, este discurso muestra un fuerte desacuerdo con las declaraciones D24: "Me interesaría vivir en un barrio netamente residencial sin variedad de equipamientos ni comercios" y D12: "Me gustaría que mi casa sea pegada a la del vecino y no tenga retiro frontal", que hacen referencia principalmente a un tipo de tejido urbano exclusivamente residencial. Además, muestra desacuerdo distintivo con las declaraciones D30: "Preferiría utilizar auto privado para desplazarme diariamente" y D19: "Preferiría un barrio con todas las calles asfaltadas destinadas al vehículo y moto exclusivamente". Tampoco está de acuerdo con D26: "Preferiría desplazarme en taxi para realizar mis actividades cotidianas", aunque esto no lo diferencia demasiado de los otros dos discursos.

Los participantes cargados en este discurso son en su mayor parte hombres, de estratos socioeconómicos medios y altos, que, si bien pueden tener disponibilidad de vehículo en el hogar, desearían no depender de ellos. Se caracterizan además por ser adultos jóvenes sin hijos. Al analizar las reflexiones finales de estos participantes, destacan expresiones que resaltan la importancia de la movilidad activa y los espacios públicos de calidad, así como características urbanas que permitan la interacción e integración social (las características de los participantes pueden observarse en la Tabla 2):

Que existan espacios de ciclovías seguros, que no solo son las rutas, también la iluminación y seguridad en varios aspectos. Que existan espacios verdes y seguros (...) conocernos entre los vecinos (...). Sí, sería en bicicleta, por el tema ecológico 
supongo, para no contaminar ni visual, ni auditivamente, ni en temas de combustión. (P02)

Yo creo que debería ser así, o sea un barrio que tenga muchas edificaciones, que permita albergar a muchísima gente; pero que, además, tenga sistemas de ciclovías, por ejemplo, que te puedas mover sin la necesidad de utilizar vehículos privados (...). Espacios verdes por montones. Me encantaría que pudiésemos llegar en bici a todos lados (...). Del centro, igual, debería ser totalmente peatonizado, por supuesto permitiendo que puedan pasar por ahí las bicicletas y los medios de transporte masivo. (P32)

Me gustaría poder salir cuando tenga que hacer algo, salir caminando o salir en bicicleta a un lugar que está cerca. No tener que coger el carro y tener que irme lejos. $\mathrm{Y}$ que tenga espacios verdes, porque creo que es bueno que haya un lugar a donde salir para estar un rato, en un parque o una orilla del río. Normalmente prefiero la bicicleta, a menos que llueva o que me esté yendo a algún lugar que esté muy lejos. Pero es porque no tengo que buscar donde parquear (...) porque es más rápido para mí, no siento el tráfico. (P30)

\section{Discurso 2: Orientados a transporte público}

En el segundo discurso detectado la movilidad sigue siendo determinante, aunque en este caso se valora las posibilidades del transporte público y comercial. Sin embargo, prevalece la expectativa de contar con garaje y jardín propio en las viviendas. En cuanto al barrio, en este discurso se prefieren características de conectividad, diversidad de usos y aceras de calidad. En tal perspectiva, la declaración de acuerdo más fuerte señala: "Me agradaría salir de mi casa y poder encontrar un taxi con facilidad" (D10). Otra declaración con puntaje positivo alto es: "Me encantaría que pasen varias líneas de bus por mi barrio" (D31); sin embargo, su error estándar indica poca consistencia en el grado de acuerdo. Por otro lado, todas las declaraciones de desacuerdo tienen un error estándar que supera el 0,4 , por lo que no son consistentes para ejemplificar el discurso. En otras palabras, el discurso F2 tiene acuerdos sobre las preferencias, pero no los tiene sobre las características no deseables relacionadas tanto a movilidad como al tejido urbano.

En este discurso se identifican participantes correspondientes a niveles socioeconómicos medios y bajos, que viven en las zonas periurbanas de la ciudad, con baja disponibilidad de vehículos en el hogar y que se trasladan cotidianamente en bus y a veces en taxi, por lo que el discurso está influenciado por la disponibilidad del modo de transporte. En su mayoría son mujeres con hijos y, a diferencia del discurso anterior, en este se identifica a varios participantes mayores de 65 años.

Varias expresiones registradas permiten resaltar la importancia que tiene para este discurso el transporte público:

(...) que pasen buses y no se demoren tanto (...). Porque, póngase, nos levantamos tarde y encima se demora media hora en venir el bus, o sea no llegamos pronto al trabajo ni nada (...). En las noches igual. Ese bus que yo tengo, solo pasa hasta las siete, de allí ya no (...). En ocasiones, cuando hace mucho sol, ya cojo taxi, o si 
llueve también (...). Claro en mi familia, toda mi familia usa bus. Mi papi, porque tiene plata, taxi. (P7)

Que tenga parques, tiendas alrededor, algo así, para compartir más con los vecinos en el parque (...). En lo que me gustaría movilizarme es igual en el bus, porque vengo más rápido, o el taxi que me cuesta más, el bus cuesta menos. (P16)

El modo de preferencia de transporte, bueno nosotros porque tenemos vehículo, pero en realidad sí utilizamos nosotros el transporte público, y aquí en donde nosotros vivimos solo existe una línea, y esa línea, si es que usted no cogió a tal hora, se quedó. Igual al regreso; entonces sí es un poco complicado. Por eso debe haber varias líneas de bus aquí. (P17)

\section{Discurso 3: Orientados a la exclusividad y autosegregación}

A diferencia de los anteriores, en este discurso sobresalen las preferencias en la tipología de la vivienda y del entorno urbano sobre la forma de movilizarse. Los participantes que se identifican con este discurso prefieren una vivienda aislada con espacio verde, con varios garajes, y en lo posible dentro de un condominio cerrado, pero con lotes individuales y casas distintas; prefieren ante todo la exclusividad. En concordancia con esto, el vehículo privado es la forma preferente y deseada de movilidad; y el transporte público es visto como un elemento negativo que no debe estar cerca del lugar de residencia. En este discurso, la declaración de acuerdo más alto es: "Para mí sería fundamental contar con garaje en mi predio" (D11). Las declaraciones de desacuerdo más importantes estuvieron relacionadas con el tipo de implantación de la vivienda: "Me gustaría que mi casa sea pegada a la del vecino y no tenga retiro frontal" (D12); "Me gustaría una casa pegada a la del vecino con un pequeño retiro frontal" (D28); "Me interesaría vivir en un conjunto habitacional cerrado con las casas iguales entre ellas" (D28). En este discurso, por lo tanto, es más importante la exclusividad de la vivienda que la forma de movilidad.

El perfil de los entrevistados que se ajustan a este discurso es el de un ciudadano de estrato medio y alto que usa el vehículo privado para su movilidad cotidiana. Generalmente vive en las afueras de la ciudad, y cuando vive en zonas céntricas le gustaría mudarse hacia zonas más periurbanas. En este discurso no se detectaron diferencias en cuanto al número de hombres y mujeres, pero resulta interesante que varios de ellos no tienen hijos, o si los tienen, ya no viven con ellos.

Al ser consultados con respecto al barrio y movilidad de preferencia, destacan testimonios como los siguientes:

Prefiero el espacio amplio antes que vivir encajonada en algo muy pequeño. Me da claustrofobia (...). Bueno, mi automóvil, porque no me gusta el transporte público (...). Los robos, los asaltos, todo. No me siento segura en el transporte público. (P11)

Prefiero un barrio alejado de la ciudad que tenga espacios verdes, que sea seguro y mi familia pueda vivir tranquila, sin preocupación de mucha delincuencia o robo o ese tipo de cosas (...). Me gustaría estar sin sonidos de carros, un poco alejado del 
ruido (...), poder tener un perro o poder jugar con los hijos. (...). El carro, porque el transporte público en Cuenca no es muy bueno y todo mundo usa carros y es más rápido (...). En carro, yo dependo de mí, a donde quiero irme y yo dispongo de mi tiempo. (P33)

\section{Discusión}

Los tres discursos identificados y explicados en el apartado anterior se obtuvieron a partir de afirmaciones relacionadas tanto con el modo de transporte como con el tejido urbano. En términos analíticos, estos discursos fueron estables al aplicar la técnica de bootstrapping, indicando un buen nivel de confiabilidad.

Los resultados seńalados tienen ciertas similitudes con trabajos previos, pero que fueron llevados a cabo en países desarrollados. De allí que la comparación no pueda realizarse más allá de las preferencias, considerando las diferencias entre esos países y el contexto latinoamericano. Por ejemplo, Jones et al. (2012) encontraron en ciudades inglesas tres discursos: santificadores de la bicicleta, los que priorizan la caminata y los adherentes al vehiculo privado. A diferencia de nuestros resultados, este estudio no detectó un discurso asociado al transporte público, debido a que se enfocó en el rol de la movilidad no motorizada en viajes personales. El estudio de Hickman y Vecia (2016) en Londres arrojó cuatro discursos: el usuario de transporte público, el ciclista comprometido, el viajero intermodal y el viajero frustrado. Es interesante el hecho de que no exista un discurso relacionado con la preferencia por el vehículo privado, contrastando con un siguiente estudio aplicado a estudiantes universitarios en Manila, esta vez por Hickman et al. (2018). En contraste con nuestro estudio, en este último caso los autores se concentraron en un grupo poblacional específico; a pesar de ello, hay una coincidencia en los discursos orientados al transporte público y a las ventajas de la exclusividad del automóvil particular. Resulta curioso que, en el caso de los estudiantes universitarios en Manila, no existan discursos vinculados con la movilidad no motorizada, lo cual demuestra -según los autores- lo complejo de la transición hacia una movilidad más sostenible en estas ciudades.

En el estudio de Borth y Summers (2018) en Edmonton (Canadá), enfocado en la relación entre el barrio y la movilidad, los autores detectaron un discurso llamado consciente de la ubicación, que destaca los valores relacionados con la movilidad activa, la accesibilidad, la proximidad y la conectividad, presentando una similitud con el discurso 1 orientados a la movilidad activa de nuestro estudio. En los resultados de Van Dijk et al. (2015), realizado con adultos mayores en Rotterdam, se destacan los discursos que apuntan, entre otras cosas, a la priorización de la accesibilidad, y guardan alguna similitud con el discurso 2 orientado al transporte público de nuestro estudio, con el que se identificaron varios adultos mayores. Sin embargo, los participantes del estudio de Rotterdam sí se orientan hacia discursos claros sobre el barrio de su preferencia, entre cuyas características se incluyen el que sean calmados, con servicios, y que permitan la socialización.

Las entrevistas con los participantes de nuestro estudio permitieron detectar algunos aspectos interesantes para la reflexión. Por ejemplo, algunas de las razones para 
preferir el uso de la bicicleta están muy asociadas a aquellas expresadas en relación con la opción por vivir en las afueras: salud, naturaleza, recreación. Es decir, los valores "salud", "naturaleza", "recreación" son vistos como positivos, pero no necesariamente propician decisiones hacia una movilidad sostenible, ya que la lejanía aumenta el uso del vehículo privado. Así también, destaca la importancia que los participantes han dado a las condiciones de "seguridad", tanto vial como personal. De las expresiones recogidas se desprende que un aumento de las condiciones de seguridad mejoraría sensiblemente el uso tanto del transporte público como de la movilidad no motorizada. Consecuentemente, las estrategias de promoción de estos modos deberán ir enfocadas a garantizar condiciones de seguridad para los ciudadanos.

\section{Conclusiones}

Este texto ha buscado contribuir al cuerpo metodológico existente en la región sobre la relación entre la movilidad y la forma urbana. Para ello se utilizó el método Q, que -tal como se detalló- permite obtener datos sobre la percepción de los sujetos respecto de una temática en particular, con una actividad más lúdica de lo que pudiese ser una encuesta, entrevista o grupo focal, ya que los participantes disfrutaron de organizar las tarjetas con las afirmaciones y colocarlas en la pirámide en su propio tiempo y sin incidencia del investigador. Para garantizar una mayor solidez de los resultados obtenidos se aplicó la técnica de bootstrapping, previamente utilizada por Zabala y Pascual (2016). No obstante, aún quedan retos pendientes para mejorar la metodología a futuro y permitir que sea más inclusiva, de tal forma que puedan participar de manera más amplia personas de estratos socioeconómicos bajos y adultos mayores.

La aplicación de la herramienta en la ciudad de Cuenca representó el reto de concretar la participación de personas de estratos socioeconómicos bajos y de adultos mayores, consecuencia de la utilización de redes sociales electrónicas para la difusión inicial de la invitación. Esta limitación fue mitigada, parcialmente, aplicando una técnica de bola de nieve a partir de contactos de los investigadores.

El estudio ha abierto nuevas cuestiones que la academia deberá abordar. Por ejemplo, será importante contrastar estos resultados con nuevos estudios en ciudades grandes latinoamericanas. Por otro lado, tomando en cuenta que tanto la movilidad como el lugar de residencia son esencialmente fenómenos geográficos y no pueden ser abstraídos de su contexto espacial, el reto de los investigadores será conocer la incidencia del medio físico en esta relación. No será igual la relación analizada en una ciudad montañosa versus una plana; o una ciudad con múltiples ríos que la atraviesan versus una sin ellos. Así también, podrá incorporarse en el futuro la variable de la condición étnica, para comprender si esto marca diferencias en las preferencias.

El estudio expuesto en este texto ha evidenciado su importancia desde dos aristas. La primera, su confirmación y reforzamiento de la riqueza de los estudios mixtos, en tanto los resultados numéricos de los análisis fueron complementados y clarificados por las expresiones vertidas por los participantes en la etapa final de la visita. En segundo lugar, este estudio resulta vanguardista en la región, ya que 
constituye el primer ejemplo de la exploración de la relación entre movilidad y forma urbana utilizando el método $Q$ en una ciudad latinoamericana. La viabilidad de la experiencia permite recomendar la aplicación de la metodología en otras ciudades de la región, adaptándola y complementándola para cada realidad. En este sentido, Dobbs et al. (2019) señalan que, para el estudio de los ecosistemas urbanos, los enfoques provenientes de los países desarrollados no deben sustituir, sino complementar, los modelos específicos de cada región.

Se espera que estudios como este contribuyan en el planteamiento de políticas públicas que favorezcan las formas urbanas más sostenibles, tomando en consideración -entre otras cosas- su influencia en la selección del modo de transporte para promover las relaciones de proximidad dentro de los límites urbanos en nuestras ciudades.

\section{Agradecimientos}

Esta publicación es parte del proyecto "Más allá del petróleo: Un estudio de la relación entre la forma urbana y el transporte en dos ciudades del Ecuador 2017 2019”, dirigido por el Grupo de Investigación LlactaLAB-Ciudades Sustentables y financiado por la Dirección de Investigaciones de la Universidad de Cuenca. Los autores agradecen a los miembros del proyecto, en especial a Daniela Astudillo y Verónica Farfán, así como a los voluntarios que participaron durante la aplicación de la herramienta de estudio.

\section{Referencias bibliográficas}

Annear, M. J., Gidlow, B. \& Cushman, G. (2009). Neighbourhood deprivation and older adults' preferences for and perceptions of active leisure participation. Annals of Leisure Research, 12(2), 96-128. https://doi.org/10.1080/11745398.2009.9686814

Blanco, J. (2016). Urbanización y movilidad: contradicciones bajo el modelo automovilintensivo. Revista Transporte y Territorio, 15, 96-113. https://www.redalyc.org/ pdf/3330/333047931007.pdf

Borth, K. \& Summers, R. (2018). Segmentation of homebuyers by location choice preferences. Housing Policy Debate, 28(3), 428-442. https://doi.org/10.1080/10511482.2017.139 3690

Capel,H. (2002). Lamorfologíadelasciudades. Tomo I: Sociedad, culturaypaisajeurbano. Ediciones del Serbal. https://play.google.com/store/books/details?id=R1ZiDAAAQBAJ

Cervero, R., Sarmiento, O. L., Jacoby, E., Gomez, L. F. \& Neiman, A. (2009). Influences of built environments on walking and cycling: lessons from Bogotá. International Journal of Sustainable Transportation, 3(4), 203-226. https://doi. org/10.1080/15568310802178314

Cheshmehzangi, A. \& Butters, C. (2016). Sustainable living and urban density: the choices are wide open. Energy Procedia, 88, 63-70. https://doi.org/10.1016/j.egypro.2016.06.020 
Cools, M., Brijs, K., Tormans, H., De Laender, J. \& Wets, G. (2012). Optimizing the implementation of policy measures through social acceptance segmentation. Transport Policy, 22, 80-87. https://doi.org/10.1016/j.tranpol.2012.05.013

Cools, M., Moons, E., Janssens, B. \& Wets, G. (2009). Shifting towards environment-friendly modes: profiling travelers using Q-methodology. Transportation, 36(4), 437-453. https://doi.org/10.1007/s11116-009-9206-z

Coppola, P., Papa, E., Angiello, G. \& Carpentieri, G. (2014). Urban form and sustainability: the case study of Rome. Procedia - Social and Behavioral Sciences, 160, 557-566. https://doi.org/10.1016/j.sbspro.2014.12.169

de Solà-Morales i Rubió, M. (1997). Las formas de crecimiento urbano. Universitat Politècnica de Catalunya.

Dobbs, C., Escobedo, F. J., Clerici, N., de la Barrera, F., Eleuterio, A. A., MacGregor-Fors, I., Reyes-Paecke, S., Vásquez, A., Zea Camaño, J. D. \& Hernández, H. J. (2019). Urban ecosystem services in Latin America: Mismatch between global concepts and regional realities? Urban Ecosystems, 22(1), 173-187. https://doi.org/10.1007/s11252-018-0805-3

Dziopa, F. \& Ahern, K. (2011). A systematic literature review of the applications of Q-Technique and its methodology. Methodology: European Journal Of Research Methods For The Behavioral \& Social Sciences, 7(2), 39-55. https://doi.org/10.1027/1614-2241/ a000021

Ewing, R. \& Cervero, R. (2010). Travel and the built environment. A meta-analysis. Journal of the American Planning Association, 76(3), 265-294. https://doi. org/10.1080/01944361003766766

Ewing, R. \& Cervero, R. (2017). "Does compact development make people drive less?” The answer is yes. Journal of the American Planning Association, 83(1), 19-25. https://doi.or g/10.1080/01944363.2016.1245112

Ewing, R. \& Hamidi, S. (2015). Compactness versus sprawl: a review of recent evidence from the United States. Journal of Planning Literature, 30(4), 413-432. https://doi. org/10.1177/0885412215595439

Figueroa, O. (2005). Transporte urbano y globalización: políticas y efectos en América Latina. Revista EURE - Revista de Estudios Urbano Regionales, 31(94), 41-53. http://dx.doi. org/10.4067/S0250-71612005009400003

Frenkel, A. \& Ashkenazi, M. (2008). Measuring urban sprawl: how can we deal with it? Environment and Planning B: Planning \& Design, 35(1), 56-79. https://doi. org/10.1068/b32155

Gutiérrez, A. (2010). Movilidad, transporte y acceso: una renovación aplicada al ordenamiento territorial. Scripta Nova, 14(331), 86. https:/www.researchgate.net/profile/Andrea_ Gutierrez31/publication/47614082_Movilidad_transporte_y_acceso_una_renovacion_ aplicada_al_ordenamiento_territorial/links/595fae5daca2728c1184d287/Movilidadtransporte-y-acceso-una-renovacion-aplicada-al-ordenamiento-territorial.pdf

Handy, S. (2017). Thoughts on the meaning of Mark Stevens's meta-analysis. Journal of the American Planning Association, 83(1), 26-28. https://doi.org/10.1080/01944363.20 16.1246379

Handy, S. L. \& Xing, Y. (2011). Factors correlated with bicycle commuting: a study in six small U.s. cities. International Journal of Sustainable Transportation, 5(2), 91-110. https:// doi.org/10.1080/15568310903514789 
Hermida, M. A., Cobo, D. \& Neira, C. (2019). Challenges and opportunities of urban fabrics for sustainable planning in Cuenca (Ecuador) - IOPscience. IOP Conf. Ser.: Earth Environ. Sci, 290. https://doi.org/10.1088/1755-1315/290/1/012118

Herold, M., Scepan, J. \& Clarke, K. C. (2002). The use of remote sensing and landscape metrics to describe structures and changes in urban land uses. Environment and Planning A: Economy \& Space, 34(8), 1443-1458. https://doi.org/10.1068/a3496

Hickman, R., Lopez, N., Cao, M., Lira, B. M. \& Biona, J. B. M. (2018). "I drive outside of peak time to avoid traffic jams - Public transport is not attractive here." Challenging discourses on travel to the university campus in Manila. Sustainability: Science Practice and Policy, 10(5), 1462. https://doi.org/10.3390/su10051462

Hickman, R. \& Vecia, G. (2016). Discourses, travel behaviour and the 'last mile' in London. Built Environment, 42(4), 539-553. https:/www.ingentaconnect.com/content/alex/ benv/2016/00000042/00000004/art00003

Huang, J., Lu, X. X. \& Sellers, J. M. (2007). A global comparative analysis of urban form: Applying spatial metrics and remote sensing. Landscape and Urban Planning, 82(4), 184-197. https://doi.org/10.1016/j.landurbplan.2007.02.010

Jones, T., Pooley, C. G., Scheldeman, G., Horton, D., Tight, M., Mullen, C., Jopson, A. \& Whiteing, A. (2012). Moving around the city: Discourses on walking and cycling in English urban areas. Environment and Planning A: Economy \& Space, 44(6), 14071424. https://doi.org/10.1068/a44387

Kasanko, M., Barredo, J. I., Lavalle, C., McCormick, N., Demicheli, L., Sagris, V. \& Brezger, A. (2006). Are European cities becoming dispersed?: A comparative analysis of 15 European urban areas. Landscape and Urban Planning, 77(1), 111-130. https://doi. org/10.1016/j.landurbplan.2005.02.003

Klinger, T., Kenworthy, J. R. \& Lanzendorf, M. (2013). Dimensions of urban mobility cultures - A comparison of German cities. Journal of Transport Geography, 31, 18-29. https:// doi.org/10.1016/j.jtrangeo.2013.05.002

Li, C.-N. \& Lai, T.-Y. (2013). An empirical analysis of links between transport and land use. Proceedings of the Institution of Civil Engineers - Transport, 166(2), 105-112. https:// doi.org/10.1680/tran.10.00043

Lynch, K. (1984, febrero 23). Good city form (The miт Press) (Reprint). The MIт Press. https:// www.amazon.com/-/es/Kevin-Lynch/dp/0262620464

Manville, M. (2017). Travel and the built environment: time for change. Journal of the American Planning Association, 83(1), 29-32. https://doi.org/10.1080/01944363.2016.1249508

Miralles-Guasch, C. (2002). Ciudad y transporte: el binomio imperfecto. Ariel. http://books. google.com/books?id=qR8wFfHovsYC\&pgis=1

Morinière, L. C. E. \& Hamza, M. (2012). Environment and mobility: a view from four discourses. Ambio, 41(8), 795-807. https://doi.org/10.1007/s13280-012-0333-y

Municipalidad de Cuenca. (2015). Plan de Movilidad y Espacios Públicos de Cuenca. I. Municipalidad de Cuenca. http://www.cuenca.gob.ec/?q=content/plan-de-movilidad

Olaru, D., Smith, B. \& Taplin, J. H. E. (2011). Residential location and transit-oriented development in a new rail corridor. Transportation Research Part A: Policy and Practice, 45(3), 219-237. https://doi.org/10.1016/j.tra.2010.12.007 
Oliveira, V. (2016). Urban morphology: an introduction to the study of the physical form of cities (The Urban Book Series). Springer. https://books.google.com.ec/books/about/ Urban_Morphology.html?id=WlveCwAAQBAJ\&printsec=frontcover\&source=kp_ read_button\&redir_esc $=y$

Palm, M., Gregor, B., Wang, H. \& McMullen, B. S. (2014). The trade-offs between population density and households' transportation-housing costs. Transport Policy, 36, 160-172. https://doi.org/10.1016/j.tranpol.2014.07.004

Rajé, F. (2007). Using Q methodology to develop more perceptive insights on transport and social inclusion. Transport Policy, 14(6), 467-477. https://doi.org/10.1016/j. tranpol.2007.04.006

R Core Team. (2016). R: A language and environment for statistical computing. R Foundation for Statistical Computing. http://www.r-project.org/

Stephenson, W. (1953). The study of behavior; Q-technique and its methodology. University of Chicago Press. https://psycnet.apa.org/record/1954-06810-000

Stevens, M. R. (2017). Does compact development make people drive less? Journal of the American Planning Association, 83(1), 7-18. https://doi.org/10.1080/01944363.201 6.1240044

Tsai, Y.-H. (2005). Quantifying urban form: compactness versus 'sprawl'. Urban Studies, 42(1), 141-161. https://doi.org/10.1080/0042098042000309748

Turok, I. (2016). Housing and the urban premium. Habitat International, 54, 234-240. https:// doi.org/10.1016/j.habitatint.2015.11.019

Van Dijk, H. M., Cramm, J. M., Van Exel, J. \& Nieboer, A. P. (2015). The ideal neighbourhood for ageing in place as perceived by frail and non-frail community-dwelling older people. Ageing \& Society, 35(8), 1771-1795. https://doi.org/10.1017/S0144686X14000622

Van Exel, J. \& De Graaf, G. (2005). Q methodology: A sneak preview. https://www.researchgate. net/profile/Gjalt_Graaf/publication/228574836_Q_Methodology_A_Sneak_ Preview/links/02bfe50f946fc9978b000000.pdf

Van Exel, N. J. A., De Graaf, G. \& Rietveld, P. (2011). I can do perfectly well without a car! Transportation, 38(3), 383-407. https://link.springer.com/article/10.1007/s11116010-9315-8

Watts, S. \& Stenner, P. (2012). Doing Q Methodological Research: theory, method and interpretation. Sage Publications. http://oro.open.ac.uk/30638/

Wheeler, S. M. (2015). Built Landscapes of metropolitan regions: an international typology. Journal of the American Planning Association, 81(3), 167-190. https://doi.org/10.1080 /01944363.2015.1081567

Zabala, A. (2014). qmethod: A Package to explore human perspectives using Q Methodology. The R Journal, 6(2), 163-173. https://doi.org/10.32614/RJ-2014-032

Zabala, A. \& Pascual, U. (2016). Bootstrapping Q Methodology to improve the understanding of human perspectives. PloS One, 11(2), e0148087. https://doi.org/10.1371/journal. pone. 0148087

Zhao, P. \& Lu, B. (2011). Managing urban growth to reduce motorised travel in Beijing: one method of creating a low-carbon city. Journal of Environmental Planning and Management, 54(7), 959-977. https://doi.org/10.1080/09640568.2010.547684 